\title{
Gait training after spinal cord injury: safety, feasibility and gait function following 8 weeks of training with the exoskeletons from Ekso Bionics
}

\author{
Carsten Bach Baunsgaard $\left(^{1}\right)^{1}$ Ulla Vig Nissen ${ }^{1}$ - Anne Katrin Brust ${ }^{2} \cdot$ Angela Frotzler $^{2} \cdot$ Cornelia Ribeill $^{3}$. \\ Yorck-Bernhard Kalke ${ }^{3}$ - Natacha León ${ }^{4}$ • Belén Gómez ${ }^{4}$ Kersti Samuelsson ${ }^{5}$ - Wolfram Antepohl ${ }^{5}$.

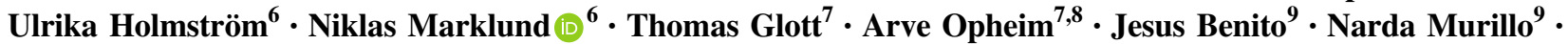 \\ Janneke Nachtegaal $^{10} \cdot$ Willemijn Faber $^{10} \cdot$ Fin Biering-Sørensen ${ }^{1}$
}

Received: 22 February 2017 / Revised: 15 August 2017 / Accepted: 15 August 2017 / Published online: 6 November 2017

(C) International Spinal Cord Society 2018

\begin{abstract}
Study design Prospective quasi-experimental study, pre- and post-design.

Objectives Assess safety, feasibility, training characteristics and changes in gait function for persons with spinal cord injury (SCI) using the robotic exoskeletons from Ekso Bionics.
\end{abstract}

Setting Nine European rehabilitation centres.

Methods Robotic exoskeleton gait training, three times weekly over 8 weeks. Time upright, time walking and steps in the device (training characteristics) were recorded longitudinally. Gait and neurological function were measured by 10 Metre Walk Test (10 MWT), Timed Up and Go (TUG), Berg Balance Scale (BBS), Walking Index for Spinal Cord Injury (WISCI) II and Lower Extremity Motor Score (LEMS).

Results Fifty-two participants completed the training protocol. Median age: 35.8 years (IQR 27.5-52.5), men/women: $N=36 / 16$, neurological level of injury: C1-L2 and severity: AIS A-D (American Spinal Injury Association Impairment Scale). Time since injury (TSI) $<1$ year, $N=25 ;>1$ year, $N=27$.

No serious adverse events occurred. Three participants dropped out following ankle swelling (overuse injury). Four participants sustained a Category II pressure ulcer at contact points with the device but completed the study and skin normalized. Training characteristics increased significantly for all subgroups. The number of participants with TSI $<1$ year and gait function increased from 20 to 56\% $(P=0.004)$ and 10MWT, TUG, BBS and LEMS results improved $(P<0.05)$. The number of participants with TSI $>1$ year and gait function, increased from 41 to $44 \%$ and TUG and BBS results improved $(P<0.05)$.

Conclusions Exoskeleton training was generally safe and feasible in a heterogeneous sample of persons with SCI. Results indicate potential benefits on gait function and balance.

Shared first authorship

Carsten Bach Baunsgaard and Ulla Vig Nissen contributed equally to this work.

Electronic supplementary material The online version of this article (https://doi.org/10.1038/s41393-017-0013-7) contains supplementary material, which is available to authorized users.

Carsten Bach Baunsgaard

cb.baunsgaard@gmail.com

carsten.bach.baunsgaard@regionh.dk

Extended author information available on the last page of the article

\section{Introduction}

Recovery of gait and related functions such as balance and mobility are important priorities for persons with spinal cord injury (SCI) $[1,2]$. Recovery of gait function is possible for some persons with an incomplete SCI [3], but less likely if the injury is complete $[4,5]$. A central paradigm in rehabilitation is task-specific training aimed at recovery of an identified and desired function [6].

Wearable robotic exoskeletons are motorized orthoses that facilitate untethered standing and walking over ground. 
These devices can support multiple step repetitions while having full weight bearing on the body thus, being taskspecific for rehabilitation of gait function.

Rationales for engaging in exoskeleton training, in cases where rehabilitation of gait function is not the aim, could be a desire to decrease time in sitting position [7], to test effects on secondary complications following SCI, such as pain, spasticity, bowel and bladder function [8-11] or use of the exoskeleton as a potential future mobility device.

Previous studies of wearable exoskeletons have investigated safety and changes in training characteristics within small study samples with an overall $75 \%$ majority of participants with complete SCI [12]. There is a need to assess robotic wearable exoskeleton gait training in the different subgroups of the heterogeneous SCI population, i.e., persons with recent and chronic injury, paraplegia and tetraplegia as well as persons with complete and incomplete SCI. There is also a need for studies representing the different models of wearable exoskeletons on the market. To date, only few studies using the exoskeletons from Ekso Bionics (Ekso Bionics, Richmond, CA) have been published $[8,10,13,14]$.

The primary objective of this study was to assess safety and feasibility during an eight week training programme with the robotic exoskeletons from Ekso Bionics for persons with SCI, while representing a broad range of participants based on severity and level of injury, as well as time since injury. Feasibility testing included documentation of the number of participants who completed the training protocol, changes in training characteristics during the exoskeleton gait training and perceived rate of exertion during training. The secondary objective was to assess changes in gait function outside of the exoskeleton in the subgroup of participants who were able to walk without the exoskeleton.

\section{Methods}

\section{Study design}

The study was designed as an open-label, prospective quasiexperimental study with a pre- and post-design. The data collection was conducted as a multicentre study across nine European SCI rehabilitation centres located in Denmark, Germany, the Netherlands, Norway, Spain, Sweden and Switzerland.

\section{Participants}

The study population was a convenience sample recruited at each of the nine centres. Eligibility criteria are listed in Table 1. Data collection was completed between April 2014 and March 2016. Recruitment was done by verbal

Table 1 Eligibility criteria

\begin{tabular}{|c|c|}
\hline Inclusion criteria & Exclusion Criteria \\
\hline $\begin{array}{l}\text { A traumatic or non-traumatic SCI with either motor complete (AIS A or B) } \\
\text { with NLI from C7 to L2 (inclusive) or motor incomplete (AIS C and D) } \\
\text { with NLI from C1 to L2 (inclusive), as determined by the ISNCSCI }{ }^{18}\end{array}$ & $\begin{array}{l}\text { Previous training with an exoskeleton and other types of robotic } \\
\text { assisted gait training }\end{array}$ \\
\hline Age $15-65$ years at time of entry to the trial (some centres $18-65$ years) & Spinal instability \\
\hline More than 30 days of time since injury (TSI) & Acute deep vein thrombosis \\
\hline $\begin{array}{l}\text { Body height } 157-188 \mathrm{~cm} \text { OR max hip width } 42 \mathrm{~cm} \text {, upper leg length } \\
51-61.4 \mathrm{~cm} \text { and lower leg length } 48-63.4 \mathrm{~cm}\end{array}$ & $\begin{array}{l}\text { Severe, recurrent attacks of autonomic dysreflexia requiring medical } \\
\text { intervention }\end{array}$ \\
\hline Maximum body weight of $100 \mathrm{~kg}$ & $\begin{array}{l}\text { Heterotopic Ossification in the lower extremities resulting in } \\
\text { restrictions of ROM at the hip or knee }\end{array}$ \\
\hline Sufficient upper extremity strength to use a front-wheeled walker & $\begin{array}{l}\text { Two or more pathological fractures in the last } 48 \text { months in a major } \\
\text { weight bearing bone in the lower extremity (femur or tibia) }\end{array}$ \\
\hline $\begin{array}{l}\text { Sufficient range of motion to achieve a reciprocal gait pattern and to } \\
\text { perform sit-to-stand transition in the device }\end{array}$ & Hip subluxation \\
\hline $\begin{array}{l}\text { Medically stable and cleared by a physician for full weight bearing } \\
\text { locomotor training }\end{array}$ & Cognitive deficits \\
\hline \multirow[t]{4}{*}{$\begin{array}{l}\text { Standing orthostatic tolerance trial by standing } 15 \text {-minute, fully supported } \\
\text { in a standing-frame, while measuring blood pressure regularly }\end{array}$} & $\begin{array}{l}\text { Spasticity assessed with the Modified Ashworth Scale of } \leq 4 \text { in } \\
\text { lower extremity muscles }\end{array}$ \\
\hline & Skin integrity issues in areas in contact with the device \\
\hline & $\begin{array}{l}\text { Concurrent neurological injury or any other issue that in the opinion } \\
\text { of the investigator would confound the results }\end{array}$ \\
\hline & Pregnancy \\
\hline
\end{tabular}

AIS American Spinal Injury Association (ASIA) impairment scale, ISNCSCI International Standards for Neurological Classification of SCI, $N L I$ neurological level of injury 
communication at in- and outpatient facilities, as well as written advertisement for the study at each centre and in consumer magazines.

The study protocol and all tests were systematically reviewed at two kick-off meetings prior to study start. All researchers and therapists involved in the study attended in order to ensure consistency in training and testing procedures.

\section{Training protocol}

This study used the two exoskeletons manufactured by Ekso Bionics, the Ekso ( $n=8$ participants) and the Ekso GT ( $n=44$ participants). Control of the walk-mode was done by the three settings Max, Fixed or Adapt. Either a frontwheeled walker or crutches were used as assistive devices. At least one Ekso-certified physiotherapist assisted and guided the participant while walking.

The training protocol consisted of gait training three times per week for eight weeks and the training protocol was considered completed if, at least, 16 out of the 24 training sessions (TS) were attended. The training intervention was given as an "add on" to existing training. The study did not control for other types of training the participants participated in.

\section{Assessments}

Training characteristics of the gait training were described by the outcome variables total up time (time standing plus time walking), walk time (time in walk motion) and number of steps, recorded by the device during the TS, alongside the walk-mode and the assistive device used. After each TS the participants' Rate of Perceived Exertion (RPE) on the Borg Scale $(6=$ very easy to $20=$ very exhaustive) [15] was recorded by asking the question: "what was your perceived exertion for the whole TS on a scale from a 6 to 20?" [16].

Skin integrity at contact points with the device was investigated before and after each TS and any adverse events were documented. Skin ulcers were categorized according to The US National Pressure Ulcer Advisory Panel and European Pressure Ulcer Advisory Panel pressure ulcer classification system [17]: Category I, Non-blanchable Erythema; Category II, Partial Thickness Skin Loss; Category III, Full Thickness Skin Loss; Category IV, Full Thickness Tissue Loss; Unstageable, Depth Unknown.

A neurological examination was performed, according to the ISNCSCI [18, 19] at baseline, at end of the training period (TS24) and at a follow-up (FU) session 4 weeks after the last TS. Lower Extremity Motor Score (LEMS) [20] was used as an outcome measure instead of the total upper and lower extremity motor score since LEMS has shown to be a better predictor of gait function than the total score [21]. SCI-subgroups were defined by three dichotomizations: Tetraplegia (neurological level of injury, NLI C1-C8) or paraplegia (NLI T1-L2), recently injured (time since injury, TSI $<1$ year) or chronically injured (TSI $\geq 1$ year), and Motor complete SCI (American Spinal Injury Association Impairment Scale, AIS A and B) or motor incomplete (AIS $\mathrm{C}$ and D).

Participants who had or acquired gait function during the training period performed the following tests: 10 Metre Walk Test (10MWT) [20, 22-24], Timed Up and Go (TUG) [22, 23], Berg Balance Scale (BBS) [25, 26] and Walking Index for Spinal Cord Injury II (WISCI II) [20, 24]. These tests were recorded at baseline, midway (TS12), at end (TS24) and at FU. The 10MWT was performed at comfortable speed and with flying start. Gait function without the exoskeleton was defined as the ability to complete the 10MWT independently without an assistant, with or without a walker or crutches and/or with a brace. Only participants with LEMS $\geq 1$ were included in the analysis of gait function.

Heart rate (HR) and blood pressure (BP) measurements were recorded in sitting position before walking and after 10 min of walking. Change from sitting to walking HR and BP was used as an outcome measure of physical strain and repeated measures were performed at TS1, TS12 and TS24 to test change over time.

\section{Statistical methods}

Repeated measures were analysed with a linear Mixed Model analysis, using compound symmetry for the repeated covariance type, group differences were assessed as a fixed factor and pairwise comparisons were adjusted with Bonferroni correction. Changes in number of persons with gait function was analysed with McNemar's test for dichotomous variables. Statistical significance was set at $\alpha$ $=0.05$. Statistical analyses were performed with IBM SPSS Statistics version 22 (IBM Corp., Released 2013. Armonk, NY, USA).

\section{Statement of ethics}

All applicable institutional and governmental regulations concerning the ethical use of human volunteers were followed during the course of this research. All the necessary approvals were obtained in each centre. All participants received oral as well as written information about the study before written consent was obtained. The study followed the Helsinki Declaration guidelines. The study was registered at ClinicalTrials.gov with identifier: NCT02132702. 
Table 2 Study population characteristics

\begin{tabular}{|c|c|c|}
\hline & Included $(n=52)$ & Excluded $(n=8)$ \\
\hline Age, median (IQR) (years) & $35.8(27.5-52.6)$ & $47(32.5-61.8)$ \\
\hline Gender & $n(\%)$ & $n(\%)$ \\
\hline Men & $36(69.2 \%)$ & $6(75 \%)$ \\
\hline Woman & $16(30.8 \%)$ & $2(25 \%)$ \\
\hline BMI, median (IQR) $\left(\mathrm{m} / \mathrm{kg}^{2}\right)$ & $24.1(22.0-26.2)$ & $22.5(21.0-24.4)$ \\
\hline Time since injury & $n(\%)$; median (IQR) (years) & $n(\%)$; median (IQR) (years) \\
\hline Recently injured (TSI $\leq 1$ year) & $25(48 \%) ; 0.3(0.2-0.4)$ & $2(25 \%) ; 0.5(0.4-0.6)$ \\
\hline Chronically injured (TSI >1 year) & $27(52 \%) ; 5.5(2.1-10.8)$ & $6(75 \%) ; 13.7(7.2-28.5)$ \\
\hline Spinal cord injury aetiology & $n(\%)$ & $n(\%)$ \\
\hline Sport/Leisure & $16(30.8 \%)$ & $1(12.5 \%)$ \\
\hline Assault & $2(3.8 \%)$ & $0(0 \%)$ \\
\hline Transport & $17(32.7 \%)$ & $2(25 \%)$ \\
\hline Fall & $7(13.5 \%)$ & $1(12.5 \%)$ \\
\hline Other traumatic cause & $1(1.9 \%)$ & $0(0 \%)$ \\
\hline Non-traumatic spinal cord dysfunction & $9(17.3 \%)$ & $4(50 \%)$ \\
\hline NLI and severity of injury at baseline & $n(\%)$ & $n(\%)$ \\
\hline C1-C4 AIS A, B, C & $0(0 \%)$ & $0(0 \%)$ \\
\hline C5-C8 AIS A, B, C & $4(7.7 \%)$ & $2(25 \%)$ \\
\hline T1-S5 AIS A, B, C & $29(55.8 \%)$ & $4(50 \%)$ \\
\hline All AIS D & $19(36.5 \%)$ & $2(25 \%)$ \\
\hline Grouping of neurological injury & $n(\%)$ & $n(\%)$ \\
\hline $\begin{array}{l}\text { Motor complete tetraplegia (C1-C8, AIS } \\
\text { A and B) }\end{array}$ & $3(5.8 \%)$ & $1(12.5 \%)$ \\
\hline $\begin{array}{l}\text { Motor incomplete tetraplegia C7-C8, AIS } \\
\text { A and B }\end{array}$ & $11(21.2 \%)$ & $1(12.5 \%)$ \\
\hline $\begin{array}{l}\text { Motor complete paraplegia T1-L2, AIS A } \\
\text { and B }\end{array}$ & $22(42.3 \%)$ & $3(37.5 \%)$ \\
\hline $\begin{array}{l}\text { Motor incomplete paraplegia T1-L2, AIS } \\
\mathrm{C} \text { and D }\end{array}$ & $16(30.8 \%)$ & $3(37.5 \%)$ \\
\hline
\end{tabular}

AIS American Spinal Injury Association Impairment Scale, BMI body mass index, IQR interquartile range, $N L I$ neurological level of injury, TSI time since injury

\section{Results}

Population characteristics are shown in Table 2 and details on centres are listed as Supplementary Material 1.

\section{Feasibility and adherence to the protocol and adverse events}

Eligibility criteria were met by 60 participants. Of these, completed 52 participants $(87 \%)$ the training protocol and 8 dropped out. Reasons for drop out were time constraint $(N=2)$, surgery not related to the training $(N=1)$, adverse events with ankle swelling $(N=3)$, and concurrent medical conditions $(N=2$, one participant had a previous minor traumatic brain injury and one had a previous minor stroke). The two concurrent medical conditions were at the screening session evaluated not to affect training or confound the results, but spasticity was too prominent during the first TS and the participants were excluded. There were no serious adverse events and no falls. The reported adverse events and skin issues are listed in Table 3.

\section{Change in training characteristics over time}

The group that adhered to the training protocol $(N=52)$ had a median number of $21(88 \%)$ completed sessions (IQR 20-23.5, range 17-24). The training characteristics up time, walk time, ratio between the two and number of steps are shown in Fig. 1a-c. All training characteristics increased significantly from TS1 to TS24 in a mixed model analysis (up time: $F=2.168, P<0.001$; walk time: $F=10.988, P<$ 0.001 ; steps: $F=15.556, P<0.001)$, including all subgroups: recently and chronically injured, paraplegia and tetraplegia and incomplete and complete injury $(P<0.001)$. There were no differences in up time between groups (no interactions, no main effects). Walk time was longer for 
Table 3 Adverse events and skin issues

Adverse events that lead to exclusion $(n=3$; chronic AIS A-B)

Swelling of the ankle joint

Occurred the day after training, unilaterally. Nothing unexpected occurred during the training session. In two of the cases, the joint effusion was preceded by pain or spasticity in the hip of the same leg after training. Standard X-rays of the 3 ankles showed no fracture and with rest the swelling disappeared.

Adverse events that did not lead to exclusion or drop out $(n=19)$

Dizziness or syncope $(n=9)$

Dizziness was reported by eight participants at one or more occasions and the session was stopped (participants NLI-AIS: C7-B, T4-A, T4-A, T9-B, T10-A, T10-A, T11-C and T12-C)

One participant experienced a syncope (T3-A) while walking.

Was helped to sit without problems. No injuries occurred.

Neurological symptoms $(n=3)$

Sensory disturbances in hand while walking with crutchesresolved by switching walker with crutches.

Mechanical errors and pain in relation to sit-to-stand $(n=5)$ :

Device error that cancelled the training $(n=2)$ and episodes during stand-to-sit procedure that caused pain around the ribs or sacrum $(n=3)$

Skin issues $(n=9)$

Blanching erythema or non-blanching Category I PU $(n=5)$

Locations thigh, tibia, instep of foot at contact points with the device or at the heal

Category II PU $(n=4)$

Location shoulder at contact point with backpack strap $(n=1)$. The strap was too tight and scratched the skin, causing a blister.

Location thigh $(n=2)$. The strap caused skin abrasion. Resolved by new straps from the manufacturer.

Location instep of the foot $(n=1)$. Contact point of foot strap. Resolved by new footwear.

AIS American Spinal Injury Association Impairment Scale, $N L I$ neurological level of injury, $P U$ pressure ulcer

chronically injured participants compared to recently injured participants with a mean difference of $5.3 \mathrm{~min}(95 \%$ confidence interval (CI) $0.8-9.8 ; P=0.22$ ), but there was no difference in the number of steps in a TS. There were no group differences between participants with paraplegia versus tetraplegia in walk time $(F=2.821, P=0.100)$, or number of steps in a session $(F=1.358, P=0.250)$. There were no differences in walk time for participants with incomplete, versus complete injuries $(F=1.532, P=$ 0.222). The participants with incomplete injuries had more steps per session than the group with complete SCI, the mean difference was 335 steps (95\% CI 112-558, $P=$ 0.004).

Figure 1e shows the distribution at each TS of participants using the two assistive devices: walker, or crutches. Figure 1f shows the distribution of participants using each of the three walk-modes: Max, Fixed and Adapt.

\section{Physical exertion during walking}

The RPE at each TS is shown in Fig. 1d. The median Borg values correspond to "light" to "somewhat hard" exertion. A mixed model analysis showed a significant decrease over time of RPE $(F=2.269, P=0.001)$. There were no significant group differences in RPE between participants with recently vs. chronically injuries $(F=0.45, P=0.833)$, participants with tetra- vs. paraplegia $(F=0.000, P=$ $0.983)$ or complete vs. incomplete SCI $(F=0.004, P=$ 0.948).

There were no significant differences over time for HR, or BP between time points TS1, TS12 and TS24 $(P>0.05)$. Within the session, HR increased 15-21\% $(P<0.001)$ from sitting to walking at the three time points but corresponding BP did not change significantly.

\section{Number of persons with gait function outside of the exoskeleton}

Figure 2 shows the changes in number of participants with gait function over time, split between recently injured and chronically injured participants. In the recently injured group, five participants (20\%) had gait function at baseline which increased to $14(56 \%)$ at TS24, (test statistic $=7.11$, $P=0.004)$ and to 15 participants $(60 \%)$ at follow up (test statistics $=0, P=1.00$ ). In the chronically injured group, 11 participants $(41 \%)$ had gait function at baseline. One chronically injured participant acquired gait function during the training period and retained this at follow up, making a total of $12(44 \%)$. The participant had a 13 years old T12 injury, AIS D and changed LEMS from 36 to 37 from screening to TS24 and at follow up.

Figure 3 shows the individual plots for the subgroup of participants who had gait function outside of the exoskeleton. Table 4 illustrate the estimated marginal means and test statistics from a mixed model analysis of the gait tests, at each time point for the same participants. The recently injured participants significantly improved TUG, 10MWT, BBS and LEMS but not WISCI II from baseline to TS24. The chronically injured participants significantly improved TUG and BBS but not 10MWT, WISCI II or LEMS from baseline to TS24. These changes were retained at follow up in both groups. Two chronically injured participants with complete SCI reported a subjective experience of improved sitting balance after the two months of training.

\section{Discussion}

This study assessed safety, feasibility, changes in training characteristics, RPE and changes in gait function of an eight week training programme with the exoskeletons from Ekso 
A

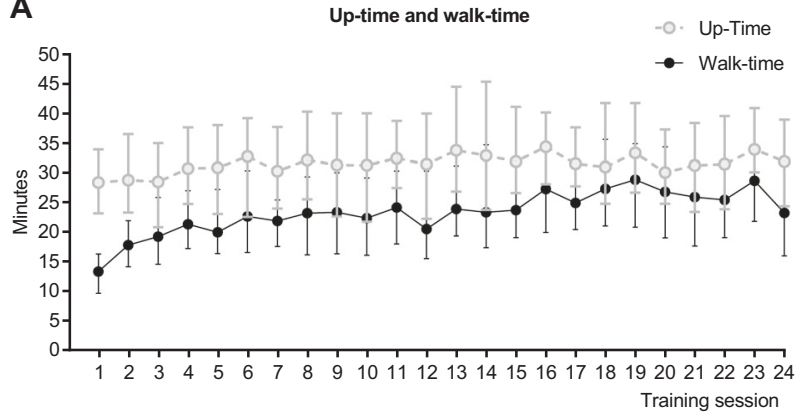

C

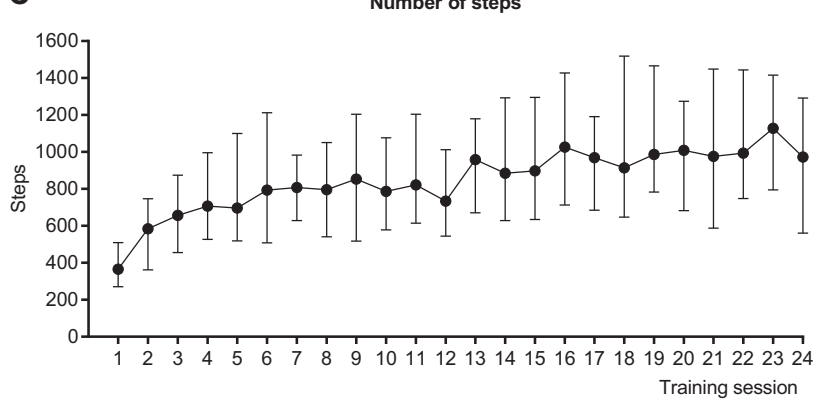

E

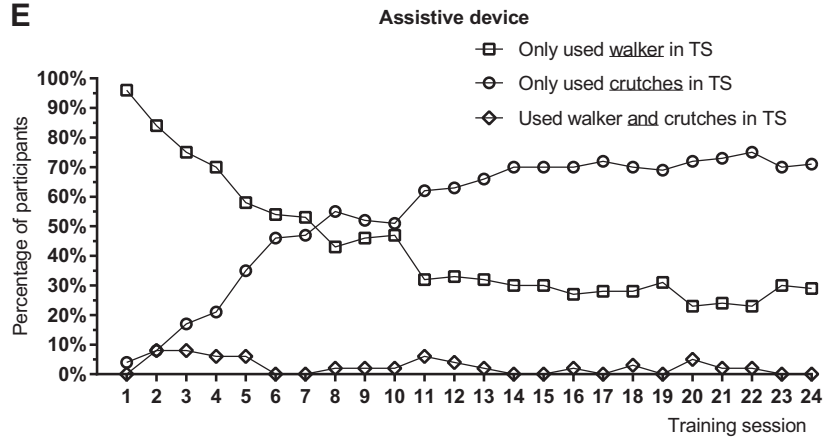

Fig. 1 Training characteristics while walking with the exoskeleton at each training session. a Median up time and walk time in minutes, b ratio of walk time and up time; $\mathbf{c}$ median number of steps; $\mathbf{d}$ Median RPE on the Borg Scale (6-20); e distribution of participants using

Bionics for persons with SCI. The study documented no serious adverse events but recorded a number of skin issues. It was feasible for all included SCI subgroups to complete the training protocol and to increase training characteristics up time, walk time and steps and, documented improvements in gait tests without the use of the exoskeleton.

\section{Safety and feasibility}

In this study, $88 \%$ of TS were completed: equivalent to 2.6 completed sessions per week, which was sufficient to lead to the progression in training characteristics for all subgroups. There was no difference in up time between SCI subgroups and only minor differences between subgroups in the outcomes of training characteristics. Transition from walker to crutches, which could reflect an improved level of
B

Walk-time/up-time ratio

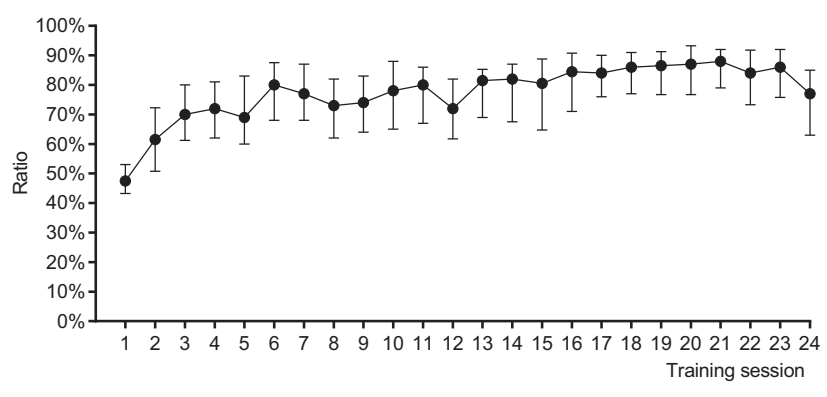

D

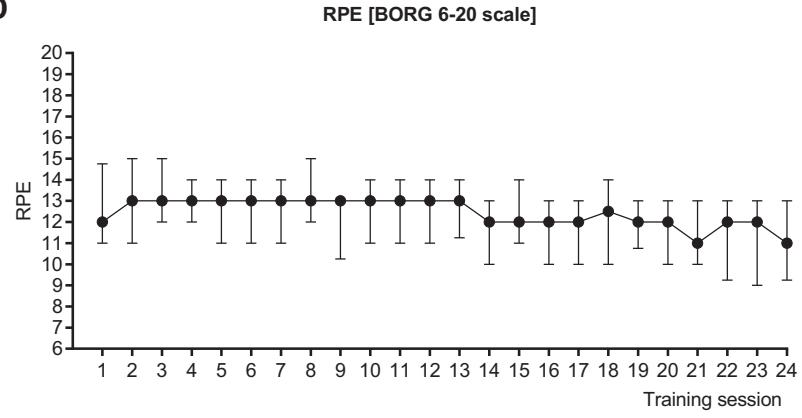

$\mathbf{F}$

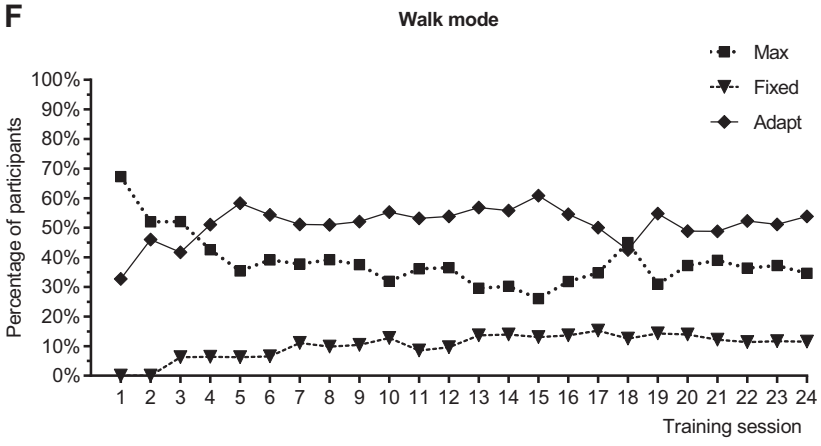

either walker or crutches as assistive devices at the training session; $\mathbf{f}$ walk-modes (Max, Fixed, Adapt). Error bars representing IQR. Abbreviations: RPE, Rate of Perceived Exertion; IQR, Interquartile range

control, happened primarily within the first four weeks of training.

Lessons to be learned were the importance of a carefully considered assessment of time consumption, the matching of expectations with the participants and close evaluation of concurrent medical conditions of participants.

The three participants who experienced a swollen joint the day following training had chronic SCI and it was concluded that the injuries were due to overuse. Benson et al. [27] reported a swollen ankle, in a study using ReWalk (Argo Medical Technologies Ltd, Yokneam Ilit, Israel), following the same pattern with joint effusion the day after training. The risk of overuse injuries should be considered when an extensive gait training programme is introduced to individuals with chronic injury who has not walked or stood up regularly for several years. A previous 
Fig. 2 Distribution of participants with and without gait function over time among recently injured $\mathbf{a}$ and chronically injured b. LEMS Lower Extremity Motor Score, $T S$ training session, TSI time since injury

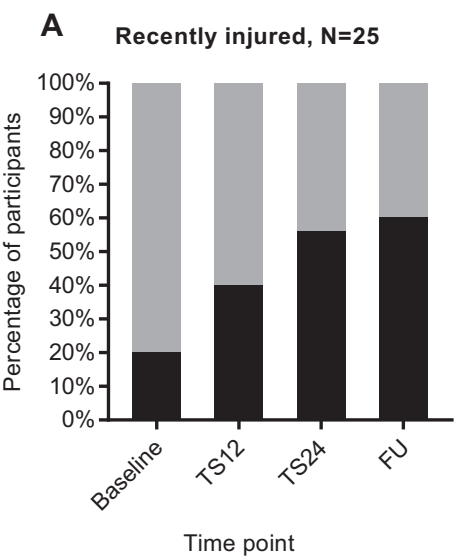

study [10] on the Ekso reported skin issues of category I in contact areas with the skin and similar findings have also been reported with the ReWalk [27].

Overall, we conclude that training with the exoskeletons from Ekso Bionics is safe, in line with the results of previous studies [12]. However, it is important that particular attention is given to prone skin.

\section{Physical strain and RPE}

Robotic exoskeletons have been designed for gait training in rehabilitation facilities, but can potentially be used as mobility devices for persons with paralysis. The devices can be seen as a further development of mechanical orthoses, such as the reciprocating gait orthosis. The daily use of these were often limited because of high energy demand and fatigue experienced after walking short distances [28] and have resulted in limited long-term use by individuals [29]. Self-perceived exertion was in the range from "fairly light" to "somewhat hard", which was supported by the relative low increase in HR measure of 15-21\% from sitting to standing. This result is also in line with previous findings of walking with an exoskeleton $[8,12]$. Interestingly, we did not find subgroup differences in RPE. The reason could be that different levels of assistance from exoskeleton were allowed. It should also be noted that the subjective Borg RPE may not be accurate in SCI [30]. However, all taken together, the results indicate that walking can be performed for an extended period of time by persons with a variety of SCI.

\section{Changes in gait function without the exoskeleton and LEMS}

The recently injured participants who had gait function without the exoskeleton improved in TUG, 10MWT, BBS and LEMS from baseline to TS24 and to follow up. Chronically injured participants improved TUG and BBS in the same periods. The significant changes from baseline to follow up indicate that these changes were sustained over time. Some degree of neurological recovery would be expected in the early phase after injury whereas this is less likely to happen in the chronic phase $[4,5,31]$. The increase seen in LEMS in the recently injured group but not in the chronically injured group could be a reflection of that.

Improvements in 10MWT, TUG and LEMS have been reported after three months of training with the exoskeleton HAL (Hybrid Assistive Limb; Cyberdyne, Inc., Japan) [32] and on 10MWT and TUG using the Lokomat [33]. The latter study [33] also found WISCI II to be less sensitive to changes in gait function in complete SCI compared to the 10MWT and TUG. The same could be suggested to be the case in our study.

The minimal clinically important difference (MCID) for the timed tests was reported by Lam et al. [34] to be $10.8 \mathrm{~s}$ (s) for the TUG test and $0.13 \mathrm{~m} / \mathrm{s}$ for the 10MWT and the minimal detectable difference (MDD) was $3.9 \mathrm{~s}$ for the TUG test and $0.05 \mathrm{~m} / \mathrm{s}$ for 10MWT. These were calculated on distribution scores measured over a three month period. Similar values were reported by $\mathrm{Wu}$ et al. [35]. In our study TUG improved by 7.0 and $7.8 \mathrm{~s}$ from baseline to TS24 for recently and chronically injured respectively. Gait speed in the $10 \mathrm{MWT}$ increased by $0.07 \mathrm{~m} / \mathrm{s}$ for both groups, based on the marginal means. These values are above MDD but below MCID. However, considering the relatively limited training period of two months and the fact that the tests were performed at comfortable speed, these results could indicate a potential for clinically relevant changes.

The improvements in the gait tests TUG and BBS in the chronically injured group could reflect improvements primarily in balance function, also supported by case reports in this study of improved sitting balance. This seems plausible, since walking with the device relies on correct weight shift from one leg to the other for initiating the next step. Training weight shift should even facilitate the training of balance skills. This could be a direction for future studies to explore. 

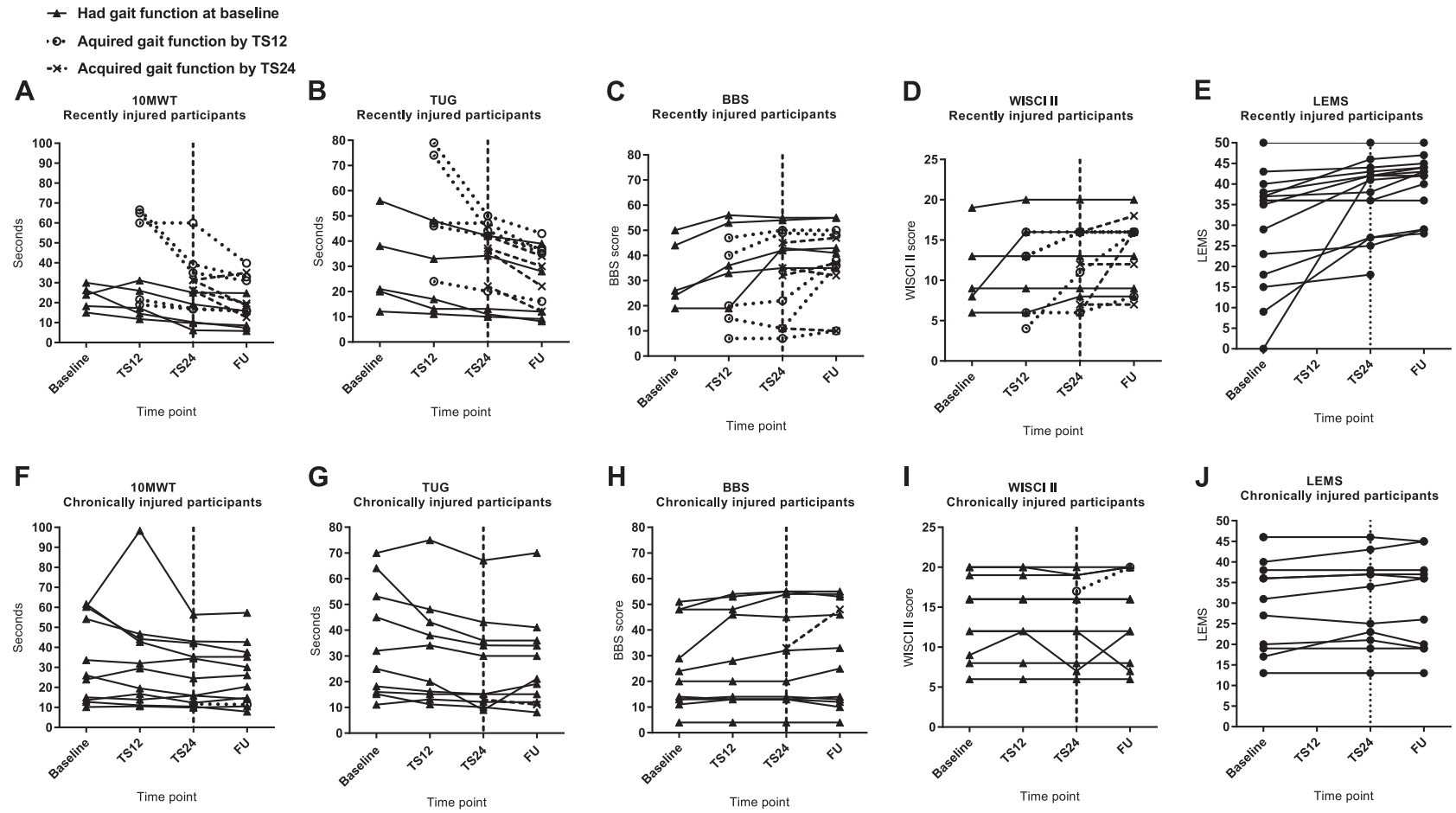

Fig. 3 Gait tests, balance tests and lower extremity motor score (LEMS) over time. Group statistics are shown in Table 4. TUG, Timed Up and Go, 10MWT, 10 Metre Walk Test, BBS, Berg Balance Scale,

\section{Strengths and limitations}

A strength of this study is that we included both participants with para- and tetraplegia, recent $(<1$ year) and chronic $(>1$ year) injuries and motor complete as well as incomplete SCI. This reflects the nature of the SCI population [36] and increases external validity. However, there is a selection bias of the study population, since the study was nonblinded and non-controlled and only participants who could commit to the training protocol were included. This would not reflect the SCI population as a whole. The heterogeneity of the study population resulted in relatively small sample sizes for the subgroups and lowered the statistical power in calculations. The differences between the two versions of devices from Ekso Bionics, the Ekso and the Ekso GT were considered minor and not to confound the results with regards to the objective of the study. Hence, both versions were allowed in the study. Participants from the age of 15 were allowed according to the protocol but none under the age of 18 were included.

To our knowledge, no other studies have tested gait function outside of the device with this or a similar over ground walking exoskeleton. However it should be stressed that the documented changes in the gait tests cannot be assigned to the exoskeleton training intervention with this current study design.
WISCI II, Walking Index for Spinal Cord Injury II, LEMS, Lower Extremity Motor Score

\section{Conclusion}

In conclusion, this multicentre study showed that the exoskeletons from Ekso Bionics is safe and feasible for use by persons with SCI, including persons with paraplegia as well as tetraplegia, recent and chronic injury as well as complete and incomplete SCI. Likewise, it was feasible for all the subgroups to increase training characteristics during training. Self-reported RPE, supported by HR measurements, was relatively low and indicates that the device can be used for longer periods of time. For those participants with gait function outside of the exoskeleton, results indicate improvements primarily in balance. This strongly supports continued research in future randomized controlled trials to test wearable exoskeleton gait training in comparison to other types of gait training.

\section{Data archiving}

All relevant data are within this manuscript and raw data are archived by the authors.

Acknowledgements We thank Linda Jones, PT, MS, Consultant, for writing the initial protocol. We would also like to thank all participants with SCI for being part of the study and to express our gratitude to the following therapists who took part in the training protocol and data collection:Mette Skov Henriksen and Mats Christer Nilfyr (Clinic for Spinal Cord Injuries, Rigshospitalet, University of Copenhagen, 
Table 4 Gait and balance tests of participants with gait function

\begin{tabular}{|c|c|c|c|c|c|c|c|c|c|c|}
\hline & & \multicolumn{4}{|c|}{ Estimated marginal means $(95 \% \mathrm{CI})$} & \multicolumn{2}{|c|}{$\begin{array}{l}\text { Univariate test } \\
\text { statistics }\end{array}$} & \multicolumn{3}{|c|}{ Pairwise comparisons ( $P$-value) } \\
\hline & & Baseline & TS12 & TS24 & Follow up & F-test & $\begin{array}{l}P- \\
\text { value }\end{array}$ & $\begin{array}{l}\text { Baseline vs. } \\
\text { TS12 }\end{array}$ & $\begin{array}{l}\text { Baseline vs. } \\
\text { TS24 }\end{array}$ & $\begin{array}{l}\text { Baseline } \\
\text { vs. FU }\end{array}$ \\
\hline \multirow[t]{5}{*}{$\begin{array}{l}\text { Recently injured } \\
(n=15)\end{array}$} & TUG [s] & $\begin{array}{l}38.3 \\
(30.5-46.1)\end{array}$ & $\begin{array}{l}36.6 \\
(29.0-44.2)\end{array}$ & $\begin{array}{l}31.3 \\
(23.8-38.8)\end{array}$ & $\begin{array}{l}28.3 \\
(20.8-35.8)\end{array}$ & 11.030 & $<0.001$ & 1.000 & 0.007 & $<0.001$ \\
\hline & $\begin{array}{l}\text { 10MWT } \\
{[\mathrm{s}]}\end{array}$ & $\begin{array}{l}35.3 \\
(26.5-44.1)\end{array}$ & $\begin{array}{l}35.8 \\
(27.1-44.4)\end{array}$ & $\begin{array}{l}28.6 \\
(20.0-37.1)\end{array}$ & $\begin{array}{l}26.0 \\
(17.5-34.4)\end{array}$ & 9.127 & $<0.001$ & 1.000 & 0.041 & 0.002 \\
\hline & BBS & $\begin{array}{l}25.4 \\
(18.2-32.5)\end{array}$ & $\begin{array}{l}28.8 \\
(21.8-35.8)\end{array}$ & $\begin{array}{l}31.5 \\
(24.6-38.4)\end{array}$ & $\begin{array}{l}33.3 \\
(26.4-40.2)\end{array}$ & 9.846 & $<0.001$ & 0.183 & 0.001 & $<0.001$ \\
\hline & WISCI II & $\begin{array}{l}12.5 \\
(10.5-14.4)\end{array}$ & $\begin{array}{l}12.6 \\
(10.7-14.6)\end{array}$ & $\begin{array}{l}12.8 \\
(11.0-14.7)\end{array}$ & $\begin{array}{l}13.7 \\
(11.8-15.5)\end{array}$ & 1.667 & 0.183 & 1.000 & 1.000 & 0.365 \\
\hline & LEMS & $\begin{array}{l}19.4 \\
(11.6-27.3)\end{array}$ & $\begin{array}{l}24.1 \\
(16.3-32.0)\end{array}$ & - & $\begin{array}{l}24.0 \\
(16.1-31.8)\end{array}$ & 5.082 & 0.01 & - & 0.022 & 0.028 \\
\hline \multirow[t]{5}{*}{$\begin{array}{l}\text { Chronically injured } \\
(n=12)\end{array}$} & TUG [s] & $\begin{array}{l}35.0 \\
(21-49.0)\end{array}$ & $\begin{array}{l}31.4 \\
(17.4-45.4)\end{array}$ & $\begin{array}{l}27.2 \\
(13.2-41.2)\end{array}$ & $\begin{array}{l}28.7 \\
(14.7-42.6)\end{array}$ & 5.558 & 0.004 & 0.555 & 0.005 & 0.028 \\
\hline & $\begin{array}{l}\text { 10MWT } \\
{[\mathrm{s}]}\end{array}$ & $\begin{array}{l}33.8 \\
(20.8-46.8)\end{array}$ & $\begin{array}{l}33.2 \\
(20.2-46.2)\end{array}$ & $\begin{array}{l}27.3 \\
(14.3-40.3)\end{array}$ & $\begin{array}{l}27.0 \\
(14.0-40.0)\end{array}$ & 2.582 & 0.072 & 1.000 & 0.322 & 0.268 \\
\hline & BBS & $\begin{array}{l}25.0 \\
(12.4-37.6)\end{array}$ & $\begin{array}{l}27.8 \\
(15.2-40.4)\end{array}$ & $\begin{array}{l}28.9 \\
(16.3-41.5)\end{array}$ & $\begin{array}{l}29.0 \\
(16.4-41.6)\end{array}$ & 4.582 & 0.009 & 0.177 & 0.021 & 0.017 \\
\hline & WISCI II & $\begin{array}{l}14.0 \\
(10.7-17.3)\end{array}$ & $\begin{array}{l}14.3 \\
(11.0-17.6)\end{array}$ & $\begin{array}{l}13.7 \\
(10.4-17.0)\end{array}$ & $\begin{array}{l}13.9 \\
(10.6-17.2)\end{array}$ & 0.466 & 0.708 & 1.000 & 1.000 & 1.000 \\
\hline & LEMS & $\begin{array}{l}14.5 \\
(7.7-21.3)\end{array}$ & $\begin{array}{l}14.8 \\
(8.0-21.6)\end{array}$ & - & $\begin{array}{l}14.7 \\
(7.8-21.5)\end{array}$ & 0.310 & 0.735 & - & 1.000 & 1.000 \\
\hline
\end{tabular}

Individual plots are shown in Fig. 3. Pairwise comparisons are Bonferroni corrected

BBS Berg Balance Scale, CI confidence interval, LEMS Lower Extremity Motor Score, TUG timed up and go, TSI time since injury, WISCI II Walking Index for Spinal Cord Injury II, 10MWT 10 metre walk test

Hornbaek, Denmark); Michael Baumberger and Ines Bersch-Porada (Swiss Paraplegic Centre (SPC), Nottwil, Switzerland); Sebastian Stallasch and Sebastian Lux (Universitäts- und Rehabilitationskliniken (RKU), Ulm, Germany); Esperanza Moreno and German Novillo (Fundación Lesionado Medular (FLM), Madrid, Spain); Niklas Fransson, Anna-Karin Hansson, Anna Granström and Mikael Lundgren (Clinical Department of Rehabilitation Medicine at Linköping University Hospital, Jönköping hospital and Västervik hospital, Sweden); Margareta Arnell and Peter Svensson (Spinal Cord Rehabilitation Unit, Uppsala University Hospital, Sweden); Ole Christian Andersen, Anna-Beth Netteland, Trygve Danielsen, Gyri S. Ingebretsen (Sunnaas Sykehus HF, Oslo, Norway); Eloy Opisso, Raquel Lopez, Josep Medina, Manel Ochoa and Eva Morales (Institut Guttmann, Neurorehabilitation Hospital, Barcelona, Spain); David Gobets, Mark van de Mijll Dekker, Ruth Sijsma (Heliomare Rehabilitation Center, Wijk aan Zee, The Netherlands). All authors were supported by their institutions' internal funds. Ekso Bionics ${ }^{\mathrm{TM}}$ provided an unconditional grant for protocol writing, salary for the clinical study lead, and expenses related to the shared database to compile data across the nine centres.

Funding Each centre was funded independently by grants or internal funds required for data collection on site. Department of Rehabilitation Medicine and Department of Medical and Health Sciences, Linköping University, Linköping, Sweden were funded by Stiftelsen Promobilia, Region Östergötland and Medical Research Council of Southeast Sweden. Spinal Cord Rehabilitation Unit, Uppsala University Hospital, Sweden was funded by Uppsala University Hospital ALF funds. Sunnaas Rehabilitation Hospital, Nesoddtangen, Norway was funded by Sunnaas Stiftelsen (The Sunnaas Foundation). Remaining centres were funded by internal funds. Ekso Bionics provided an unconditional grant for protocol writing, salary for the clinical study lead, and expenses related to the shared database to compile data across the nine centres.

\section{Compliance with ethical standards}

Conflict of interest The authors declare that they have no competing interests.

\section{References}

1 Kirshblum SC, Priebe MM, Ho CH, Scelza WM, Chiodo AE, Wuermser L-A. Spinal Cord Injury Medicine. 3. Rehabilitation Phase After Acute Spinal Cord Injury. Arch Phys Med Rehabil. 2007;88:S62-70.

2 Ditunno PL, Patrick M, Stineman M, Ditunno JF. Who wants to walk? Preferences for recovery after SCI: a longitudinal and crosssectional study. Spinal Cord. 2008;46:500-6.

3 Burns SP, Golding DG, Rolle WA, Graziani V, Ditunno JF. Recovery of ambulation in motor-incomplete tetraplegia. Arch Phys Med Rehabil. 1997;78:1169-72.

4 Mehrholz J, Kugler J, Pohl M. Locomotor training for walking after spinal cord injury. Cochrane Database Syst Rev. 2012;11: CD006676.

5 Waters RL, Yakura JS, Adkins RH, Sie I. Recovery following complete paraplegia. Arch Phys Med Rehabil. 1992;73:784-9.

6 Van Kammen K, Boonstra A, Reinders-Messelink H, den Otter R. The Combined Effects of Body Weight Support and Gait Speed on 
Gait Related Muscle Activity: A Comparison between Walking in the Lokomat Exoskeleton and Regular Treadmill Walking. PLoS ONE. 2014;9:e107323.

7 Stamatakis E, Rogers K, Ding D, Berrigan D, Chau J, Hamer M, et al. All-cause mortality effects of replacing sedentary time with physical activity and sleeping using an isotemporal substitution model: a prospective study of 201,129 mid-aged and older adults. Int J Behav Nutr Phys Acta. 2015;12:121.

8 Kozlowski AJ, Bryce TN, Dijkers MP. Time and effort required by persons with spinal cord injury to learn to use a powered exoskeleton for assisted walking. Top Spinal Cord Inj Rehabil. 2015;21:110-21.

9 Zeilig G, Weingarden H, Zwecker M, Dudkiewicz I, Bloch A, Esquenazi A. Safety and tolerance of the ReWalk exoskeleton suit for ambulation by people with complete spinal cord injury: a pilot study. J Spinal Cord Med. 2012;35:96-101.

10 Kolakowsky-Hayner SA. Safety and Feasibility of using the EksoTM Bionic Exoskeleton to Aid Ambulation after Spinal Cord Injury. J Spine 2013. https://doi.org/10.4172/2165-7939.S4-003

11 Esquenazi A, Talaty M, Packel A, Saulino M. The ReWalk powered exoskeleton to restore ambulatory function to individuals with thoracic-level motor-complete spinal cord injury. Am J Phys Med Rehabil Assoc Acad Physiatr. 2012;91:911-21.

12 Miller LE, Zimmermann AK, Herbert WG. Clinical effectiveness and safety of powered exoskeleton-assisted walking in patients with spinal cord injury: systematic review with meta-analysis. Med Devices Auckl NZ. 2016;9:455-66.

13 Sale P, Russo EF, Russo M, Masiero S, Piccione F, Calabrò RS, et al. Effects on mobility training and de-adaptations in subjects with Spinal Cord Injury due to a Wearable Robot: a preliminary report. BMC Neurol. 2016;16:12.

14 Kressler J, Thomas CK, Field-Fote EC, Sanchez J, WiderströmNoga E, Cilien DC, et al. Understanding Therapeutic Benefits of Overground Bionic Ambulation: Exploratory Case Series in Persons With Chronic, Complete Spinal Cord Injury. Arch Phys Med Rehabil. 2014;95:1878-87.

15 Borg GA. Psychophysical bases of perceived exertion. Med Sci Sports Exerc. 1982;14:377-81.

16 Anon. Perceived Exertion (Borg Rating of Perceived Exertion Scale) I Physical Activity I CDC. https://www.cdc.gov/physicala ctivity/basics/measuring/exertion.htm (accessed 26 Apr2017).

17 National Pressure Ulcer Advisory Panel, European Pressure Ulcer Advisory Panel and Pan Pacific Pressure Injury Alliance. Prevention and Treatment of Pressure Ulcers: Quick Reference Guide. Emily Haesler. Osborne Park, Western Australia: Cambridge Media; 2014. Available at: http://www.npuap.org/wp-content/ uploads/2014/08/Quick-Reference-Guide-DIGITAL-NPUAPEPUAP-PPPIA-Jan2016.pdf (accessed on 15 Aug 2016).

18 Kirshblum SC, Burns SP, Biering-Sorensen F, Donovan W, Graves DE, Jha A, et al. International standards for neurological classification of spinal cord injury (revised 2011). J Spinal Cord Med. 2011;34:535-46.

19 Itzkovich M, Gelernter I, Biering-Sorensen F, Weeks C, Laramee MT, Craven BC, et al. The Spinal Cord Independence Measure (SCIM) version III: reliability and validity in a multi-center international study. Disabil Rehabil. 2007;29:1926-33.
20 Alexander MS, Anderson K, Biering-Sorensen F, Blight AR, Brannon R, Bryce T, et al. Outcome Measures in Spinal Cord Injury. Spinal Cord. 2009;47:582-91.

21 Marino RJ, Graves DE. Metric properties of the ASIA motor score: subscales improve correlation with functional activities. Arch Phys Med Rehabil. 2004;85:1804-10.

22 Poncumhak P, Saengsuwan J, Kamruecha W, Amatachaya S. Reliability and validity of three functional tests in ambulatory patients with spinal cord injury. Spinal Cord. 2013;51:214-7.

23 van Hedel HJ, Wirz M, Dietz V. Assessing walking ability in subjects with spinal cord injury: validity and reliability of 3 walking tests. Arch Phys Med Rehabil. 2005;86:190-6.

24 Jackson AB, Carnel CT, Ditunno JF, Read MS, Boninger ML, Schmeler MR, et al. Outcome measures for gait and ambulation in the spinal cord injury population. J Spinal Cord Med. 2008;31:487-99.

25 Wirz M, Müller R, Bastiaenen C. Falls in persons with spinal cord injury: validity and reliability of the Berg Balance Scale. Neurorehabil Neural Repair. 2010;24:70-7.

26 Lemay J-F, Nadeau S. Standing balance assessment in ASIA D paraplegic and tetraplegic participants: concurrent validity of the Berg Balance Scale. Spinal Cord. 2010;48:245-50.

27 Benson I, Hart K, Tussler D, Middendorp van JJ. Lower-limb exoskeletons for individuals with chronic spinal cord injury: findings from a feasibility study. Clin Rehabil. 2016;30:73-84.

28 Bernardi M, Canale I, Castellano V, Di Filippo L, Felici F, Marchetti M. The efficiency of walking of paraplegic patients using a reciprocating gait orthosis. Paraplegia. 1995;33:409-15.

29 Sykes L, Edwards J, Powell ES, Ross ER. The reciprocating gait orthosis: long-term usage patterns. Arch Phys Med Rehabil. 1995;76:779-83.

30 Lewis JE, Nash MS, Hamm LF, Martins SC, Groah SL. The relationship between perceived exertion and physiologic indicators of stress during graded arm exercise in persons with spinal cord injuries. Arch Phys Med Rehabil. 2007;88:1205-11.

31 Waters RL, Adkins RH, Yakura JS, Sie I. Motor and sensory recovery following incomplete tetraplegia. Arch Phys Med Rehabil. 1994;75:306-11.

32 Aach M, Cruciger O, Sczesny-Kaiser M, Höffken O, Meindl RC, Tegenthoff M, et al. Voluntary driven exoskeleton as a new tool for rehabilitation in chronic spinal cord injury: a pilot study. Spine J Off J North Am Spine Soc. 2014;14:2847-53.

33 Wirz M, Zemon DH, Rupp R, Scheel A, Colombo G, Dietz V, et al. Effectiveness of automated locomotor training in patients with chronic incomplete spinal cord injury: A multicenter trial. Arch Phys Med Rehabil. 2005;86:672-80.

34 Lam T, Noonan VK, Eng JJ. A systematic review of functional ambulation outcome measures in spinal cord injury. Spinal Cord. 2008;46:246-54.

$35 \mathrm{Wu}$ X, Liu J, Tanadini LG, Lammertse DP, Blight AR, Kramer JLK, et al. Challenges for defining minimal clinically important difference (MCID) after spinal cord injury. Spinal Cord. 2015;53:84-91.

36 Ijzerman MJ, Baardman G, Hermens HJ, Veltink PH, Boom HBK, Zilvold G. Comparative trials on hybrid walking systems for people with paraplegia: An analysis of study methodology. Prosthet Orthot Int. 1999;23:260-73.

\section{Affiliations}

Carsten Bach Baunsgaard $\mathbb{D D}^{1} \cdot$ Ulla Vig Nissen ${ }^{1} \cdot$ Anne Katrin Brust $^{2} \cdot$ Angela Frotzler $^{2}$ - Cornelia Ribeill ${ }^{3} \cdot$ Yorck-Bernhard Kalke $^{3}$ • Natacha León ${ }^{4} \cdot$ Belén Gómez $^{4} \cdot$ Kersti Samuelsson $^{5}$ - Wolfram Antepohl ${ }^{5} \cdot$ Ulrika Holmström $^{6} \cdot$ Niklas Marklund (iD $^{6} \cdot$ Thomas Glott $^{7}$ • Arve Opheim $^{7,8} \cdot$ Jesus Benito $^{9} \cdot$ Narda Murillo $^{9} \cdot$ Janneke Nachtegaal $^{10} \cdot$ Willemijn Faber $^{10} \cdot$ Fin Biering-Sørensen $^{1}$ 
1 Clinic for Spinal Cord Injuries, Rigshospitalet, University of Copenhagen, Copenhagen, Denmark

2 Swiss Paraplegic Centre (SPC), Nottwil, Switzerland

3 SCI Centre Orthopaedic Department Ulm University, Ulm, Germany

4 Fundación Lesionado Medular (FLM), Madrid, Spain

5 Department of Rehabilitation Medicine and Department of Medical and Health Sciences, Linköping University, Linköping, Sweden
6 Spinal Cord Rehabilitation Unit, Uppsala University Hospital, Uppsala, Sweden

7 Sunnaas Rehabilitation Hospital, Nesoddtangen, Norway

8 Rehabilitation Medicine, Institute of Neuroscience and Physiology, Sahlgrenska Academy, University of Gothenburg, Gothenburg, Sweden

9 Institute Guttmann, Neurorehabilitation Hospital, Barcelona, Spain

10 Heliomare Rehabilitation Center, Wijk aan Zee, The Netherlands 